\title{
Quality of life and fear of cancer recurrence in T1 colorectal cancer patients treated with endoscopic or surgical tumor resection
}

\author{
Hao Dang, BSc, ${ }^{1}$ Wouter H. de Vos tot Nederveen Cappel, MD, PhD, ${ }^{2}$ Sarita M. S. van der Zwaan, BSc, ${ }^{1}$ \\ M. Elske van den Akker-van Marle, PhD, ${ }^{3}$ Henderik L. van Westreenen, MD, PhD, ${ }^{4}$ Yara Backes, MD, PhD, ${ }^{5}$ \\ Leon M. G. Moons, MD, PhD, ${ }^{5}$ Fabian A. Holman, MD, PhD, ${ }^{6}$ Koen C. M. J. Peeters, MD, PhD, ${ }^{6}$ \\ Jolein van der Kraan, MD, ${ }^{1}$ Alexandra M. J. Langers, MD, PhD, ${ }^{1}$ Willem M. Lijfering, PhD, \\ James C. H. Hardwick, MD, PhD, ${ }^{1}$ Jurjen J. Boonstra, MD, PhD ${ }^{1}$
}

Leiden, Zwolle, Utrecht, The Netherlands

\begin{abstract}
Background and Aims: To optimize therapeutic decision-making in early invasive colorectal cancer (T1 CRC) patients, it is important to elicit the patient's perspective next to considering medical outcome. Because empirical data on patient-reported impact of different treatment options are lacking, we evaluated patients' quality of life, perceived time to recovery, and fear of cancer recurrence after endoscopic or surgical treatment for T1 CRC.

Methods: In this cross-sectional study, we selected patients with histologically confirmed T1 CRC who participated in the Dutch Bowel Cancer Screening Programme and received endoscopic or surgical treatment between January 2014 and July 2017. Quality of life was measured using the European Organization for Research and Treatment 30-item Core Quality of Life Questionnaire and the 5-level EuroQoL 5-dimension questionnaire. We used the Cancer Worry Scale (CWS) to evaluate patients' fear of cancer recurrence. A question on perceived time to recovery after treatment was also included in the set of questionnaires sent to patients.
\end{abstract}

Results: Of all 119 eligible patients, 92.4\% responded to the questionnaire (endoscopy group, 55/62; surgery group, 55/57). Compared with the surgery group, perceived time to recovery was on average 3 months shorter in endoscopically treated patients after adjustment for confounders (19.9 days vs 111.3 days; $P=.001)$. The 2 treatment groups were comparable with regard to global quality of life, functioning domains, and symptom severity scores. Moreover, patients in the endoscopy group did not report more fear of cancer recurrence than those in the surgery group (CWS score, $0-40$; endoscopy 7.6 vs surgery $9.7 ; P=.140$ ).

Conclusions: From the patient's perspective, endoscopic treatment provides a quicker recovery than surgery, without provoking more fear of cancer recurrence or any deterioration in quality of life. These results contribute to the shared therapeutic decision-making process of clinicians and T1 CRC patients. (Gastrointest Endosc 2019;89:533-44.)

With the introduction of population-based screening programs in several countries, the proportion of patients diagnosed with early invasive colorectal cancer (T1 CRC) has vastly increased. ${ }^{1}$ Currently, therapeutic decisionmaking for these patients is mainly based on oncologic

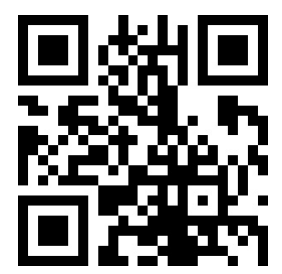

Use your mobile device to scan this QR code and watch the author interview. Download a free $Q R$ code scanner by searching "QR Scanner" in your mobile device's app store. outcomes and the risk of treatment-related adverse events. ${ }^{2}$ Because cancer care has shifted from a diseasebased to a patient-centered approach, more attention is being paid to functional outcomes and impact on quality of life (QoL) of different treatment modalities. ${ }^{3}$

Approximately $6 \%$ to $12 \%$ of patients with T1 CRC have lymph node metastases (LNM) at the time of diagnosis. ${ }^{4-6}$ These patients benefit from surgical treatment, because surgery allows adequate lymph node dissection. Histologic high-risk features associated with the presence of LNM include deep submucosal invasion (Haggitt level 4 or Kikuchi level Sm2 or 3), positive resection margins, poor differentiation, presence of lymphatic or 
vascular invasion, and tumor budding. ${ }^{7-9}$ However, in the absence of these features, T1 CRCs carry a relatively low risk of LNM (.8\%). ${ }^{8}$ In these cases, endoscopic resection is considered to be sufficient because the LNM risk does not outweigh the risk of adverse events after surgical resection.

When the aforementioned histologic criteria are used, it remains difficult to accurately predict the presence of LNM. Negative lymph nodes are found in about $80 \%$ of all patients referred for surgical resection, indicating that surgery has not provided additional oncologic benefit in these cases. ${ }^{7}$ In addition, colorectal surgery involves a significant risk of morbidity (30\%) and even mortality (1\%-5\%), especially in the elderly population, ${ }^{10-12}$ the main target group of screening programs. ${ }^{13,14}$ On the contrary, therapeutic colonoscopies, including endoscopic resection of large colon polyps, ${ }^{15,16}$ carry a low risk of morbidity and mortality and are therefore considered to be safe in the elderly. ${ }^{17}$ Because an accurate weighing up of oncologic benefit versus the risk of treatmentrelated adverse events remains challenging, eliciting the patient's perspective becomes especially important. In other words, optimal decision-making in T1 CRC patients involves balancing the aforementioned factors as well as considering relevant patient-reported outcomes such as QoL, fear of cancer recurrence, and perceived time to recovery.

Although many studies have evaluated the QoL of patients after surgery for CRC, ${ }^{18-25}$ little is known about the impact of endoscopic tumor resection on QoL. It is often assumed that endoscopic treatment results in a better overall QoL than colorectal surgery because endoscopy is less invasive and preserves the functional integrity of the colon or rectum. ${ }^{26,27}$ However, endoscopic treatment requires a more intensive follow-up program. ${ }^{28}$ This may provoke more fear of cancer recurrence and adversely influence QoL because patients are more frequently confronted with the risk of cancer recurrence. To the best of our knowledge, simultaneous evaluation of QoL, patients' perceived time to recovery, and fear of cancer recurrence after endoscopic or surgical treatment for $\mathrm{T} 1$ $\mathrm{CRC}$ has not been reported yet. Therefore, the aim of our study was to compare the QoL, perceived time to recovery, and fear of cancer recurrence between endoscopically and surgically treated T1 CRC patients.

\section{METHODS}

\section{Patients}

In this cross-sectional, 2-center study, patients were selected according to the following inclusion criteria: (1) participated in the Dutch Bowel Cancer Screening Programme, (2) diagnosed with T1 CRC (defined as histologic tumor invasion through the muscularis mucosa and into, but not beyond, the submucosa), (3) received either endo- scopic or surgical treatment at Leiden University Medical Centre or Isala Hospital, Zwolle between January 1, 2014 and August 1, 2017, and (4) provided written informed consent. Exclusion criteria were distant metastasis at time of diagnosis, synchronous colorectal carcinomas elsewhere, tumor down-staging by neoadjuvant therapy, and unable to read Dutch. The study protocol was approved by the Medical Ethical Committees of the 2 participating centers (Leiden University Medical Centre [P16.269] and Isala Hospital, Zwolle [170917]).

\section{Clinical characteristics}

Clinical baseline characteristics (sex, age, comorbidity, body mass index, prior abdominal surgery) and clinical data (tumor size, morphology, and location; treatmentrelated parameters; histology of the resected specimens; endoscopic and imaging follow-up) were retrieved from medical records. Patients' family history of CRC as well as data on marital and employment status were collected through self-reporting questionnaires. Patient comorbidity was quantified using the Charlson Comorbidity Index ${ }^{29}$ and the American Society of Anesthesiologists physical status classification system.

For the endoscopically treated group, treatment-related parameters were indication for endoscopic treatment, method of resection, and treatment-related adverse events. For the group that underwent surgery, treatment-related parameters were type of surgery, number of retrieved lymph nodes, use of colostomy, indication for surgery, whether or not prior endoscopic resection was performed, and treatment-related adverse events. Patients treated with transanal endoscopic microsurgery (TEM) were classified as endoscopically treated patients, because only a local tumor excision without lymph node dissection was performed.

\section{QoL questionnaires}

Global QoL status was measured using the validated Dutch version of the 5-level EuroQoL 5-dimension questionnaire (EQ-5D-5L). ${ }^{30,31}$ The first part of this instrument is a 5-item descriptive system (EQ-5D) representing the "societal value" of the patient's current health state. This part describes generic QoL based on 5 dimensions of health (mobility, self-care, usual activities, pain/discomfort, and anxiety/depression) with responses at 5 levels (no problems, slight problems, moderate problems, severe problems, and unable to/extreme problems). The index score, which is expressed on a scale from 0 (death) to 1 (perfect health), is calculated by matching the pattern of the 5 responses against a specific set of utilities derived from the Dutch population. ${ }^{31}$ The second part of the EQ-5D-5L represents the patient's perspective and consists of a EuroQoL selfrated visual analogue scale (EQ-VAS) ranging from 0 (worst imaginable health state) to 100 (best imaginable health state). 
We evaluated disease-specific QoL with the validated Dutch version of the European Organization for Research and Treatment 30-item Core Quality of Life Questionnaire (EORTC QLQ-C30). ${ }^{32}$ This cancer-specific questionnaire uses a 1-week time frame and includes 30 items that are linearly converted into a global health status score, 5 functional scales (physical, role, emotional, cognitive, and social functioning), 3 symptom scales (fatigue, nausea and vomiting, and pain), and 6 individual symptom scores (dyspnea, insomnia, appetite loss, constipation, diarrhea, and financial difficulties). The scores range from 0 to 100 . A higher score on the functional scales and the global health status indicates a better QoL, whereas a higher score on the symptom scales indicates a higher severity of symptoms.

\section{Fear of cancer recurrence}

We measured fear of cancer recurrence using the Dutch translation of the validated Cancer Worry Scale (CWS). ${ }^{33-36}$ This instrument evaluates patients' judged risk of cancer recurrence, the frequency and degree of their worry, and the impact of these concerns on daily functioning. The CWS, which does not use a specific time frame, consists of 4 questions with a 10-point Likert scale. These answer scores are summed to calculate the overall CWS score, which ranges from 0 to 40 . A higher score indicates more fear of cancer recurrence.

\section{Subjective time to recovery}

In both treatment groups, the patient's perceived time to recovery was evaluated with the following question: "How long after the treatment for the malignant polyp did you feel completely recovered?" Patients were requested to indicate this period with an exact amount of days, weeks, or months.

\section{Procedure}

In each participating center, all questionnaires were sent once and on the same date to the patients' home addresses along with a stamped return envelope. Patients who did not return the questionnaire within 2 weeks were contacted by phone to confirm the receipt of the questionnaire and to check whether they were still willing to participate.

\section{Statistical analysis}

Nominal and ordinal variables were expressed as frequencies and percentages and continuous variables as means and standard deviations. The Pearson $\chi^{2}$ or the Fisher exact test was used to compare categorical data, as appropriate. Continuous variables were compared using the Mann-Whitney U test.

We used multivariate linear regression to compare the EQ-5D-5L, EORTC QLQ-C30, and CWS scores of endoscopically and surgically treated patients. The analyses were adjusted for confounding variables, which were selected according to the criteria of Rothman. ${ }^{37}$ The following variables were identified in the literature as confounders: age and sex, ${ }^{2,38}$ body mass index and comorbidity, ${ }^{2,39}$ and family history of CRC. ${ }^{2,40}$ Because the American Society of Anesthesiologists physical status classification is mainly designed for the preoperative setting, ${ }^{41}$ we chose to include the Charlson Comorbidity Index in the regression analyses as an indicator for patient comorbidity.

Furthermore, to explore the short-term and long-term effects on the study outcomes of both treatments, we stratified the aforementioned analyses on the time between treatment date and moment of filling in the questionnaire. The median time of the whole study population was used as an a priori cut-off for division into 2 groups (short and long term) to create treatment groups of comparable size and to minimalize power reduction of these subgroup analyses.

Additionally, another subgroup analysis was conducted to investigate the influence of timing of surgery (ie, whether surgery was performed as primary treatment or performed after endoscopic resection) on the study outcomes. We split up the surgery group into primarily and secondarily operated patients and separately compared them with endoscopically treated patients. Because TEM may have greater impact on functional outcomes than other endoscopic resection methods, ${ }^{42}$ we also performed a sensitivity analysis with all TEM patients in the surgery group.

All statistical analyses were performed using IBM SPSS statistics version 23.0 (Chicago, Ill). $P<.05$ was considered statistically significant.

\section{RESULTS}

In total, 119 patients fulfilled our selection criteria and thus received a questionnaire (Fig. 1). Of these patients, 62 (52.1\%) were treated only with an endoscopic resection. In the surgery group ( $\mathrm{n}=57), 29$ patients were primarily treated with surgery, whereas 28 patients underwent an additional surgical resection after endoscopic treatment. The response rate was $88.7 \%(55 / 62)$ in the endoscopy group and $96.5 \%(55 / 57)$ in the surgery group. In both treatment groups, nonresponders did not give a reason for not participating in this study. There were no clinically relevant differences between responders and nonresponders (data not shown, available on request).

With regard to demographic characteristics of the patients who responded, no significant differences were found between the endoscopic and surgical treatment groups (Table 1). Because the Dutch Bowel Cancer Screening Programme exclusively targets people between 55 and 75 years old, both groups mainly consisted of elderly, retired people.

Clinical and tumor characteristics are shown in Tables 2 and 3, respectively. Figure 2 shows an overview of the therapeutic decision-making of all 


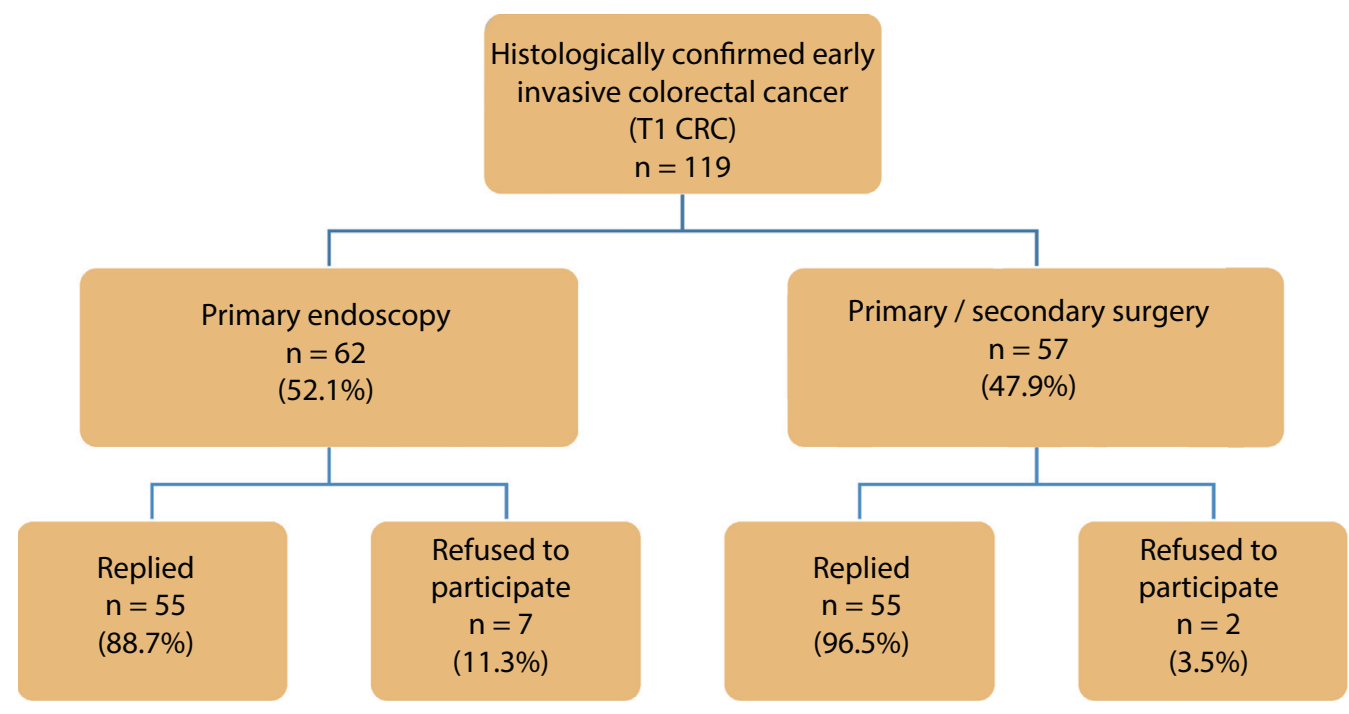

Figure 1. Flowchart of patient selection in the 2 participating centers.

included patients. In the endoscopy group, 6 patients received additional local treatment of the scar because of suspected incomplete tumor removal. The median time between primary and secondary endoscopic resection was 49.5 days (interquartile range, 34-80). Endoscopy-related adverse events mainly included postprocedural bleeding $(n=5)$ or postpolypectomy coagulation syndrome $(n=2)$. Six patients actively rejected surgery even though the treating clinician recommended an additional surgical resection. The median interval between endoscopic resection and secondary surgery was 55 days (interquartile range, 40-78). Reasons for secondary referral to the surgeon were the presence of high-risk histologic features $(\mathrm{n}=14)$ or suspected incomplete endoscopic tumor removal $(n=13)$. None of the surgically treated patients had LNM. Of all $12 \mathrm{pa}$ tients with postoperative adverse events, 3 underwent surgery again because of fascial dehiscence $(\mathrm{n}=1)$, anastomotic stricture $(\mathrm{n}=1)$, or leakage $(\mathrm{n}=1)$. The patient with an anastomotic stricture also received a permanent stoma after the reoperation. None of the patients had recurrent disease at time of filling in the questionnaire.

Figures 3 and 4 show that the intervals between treatment and moment of filling in the questionnaire were comparable in the 2 treatment groups (endoscopy, median 18 months, vs surgery, median 21 months; $P=$ .157). The median duration of this interval for the whole study population was 19 months. Using this cut-off value, we divided all patients into a short-term (endoscopy, $\mathrm{n}=28$; surgery, $\mathrm{n}=24$ ) and long-term (endoscopy, $\mathrm{n}=27$; surgery, $\mathrm{n}=31$ ) stratum.

\section{Global QoL, functioning, and symptom scores}

According to the EQ-5D-5L, generic QoL from both the patients' and societal perspective was not signifi- cantly different between the 2 treatment groups (Table 4). The disease-specific EORTC QLQ-C30 also did not show any difference in global health status and general functioning. Furthermore, symptom scores were comparable between the surgery and endoscopy groups (Table 5). The subgroup analysis with the separated surgery groups did not yield any differences in all aforementioned outcomes (data not shown; available on request). Similar results were also found in stratified analyses (data not shown; available on request), except for the short-term EQ-5D utility score (endoscopy, .89, vs surgery, .93; $P=.034$ ).

\section{Fear of cancer recurrence}

The results of the CWS are depicted in Table 6 . Endoscopically treated patients did not report more fear of cancer recurrence than surgically treated ones (endoscopy, 7.6, vs surgery, 9.7; $P=.140$ ). Stratification on time between treatment and moment of measurement showed that short-term (endoscopy, 7.9, vs surgery, 9.4; $P=.606$ ) and long-term (endoscopy, 7.2, vs surgery, 10.0; $P=.192$ ) fear of cancer recurrence also did not significantly differ between the treatment groups. However, subgroup analysis showed that when compared with the endoscopy group, primarily operated patients reported significantly more fear of cancer recurrence (endoscopy, 7.6, vs primary surgery, 10.9; $P=$ $.025)$ but secondarily operated patients did not (endoscopy, 7.6, vs secondary surgery, $8.5 ; P=.789$ ).

\section{Subjective time to recovery}

Responses to the question about the perceived time to recovery showed that recovery in endoscopically treated patients was 3 months faster than surgically treated patients. With correction for all confounding variables, this difference was strongly significant $(P=.001)$. This result was not 


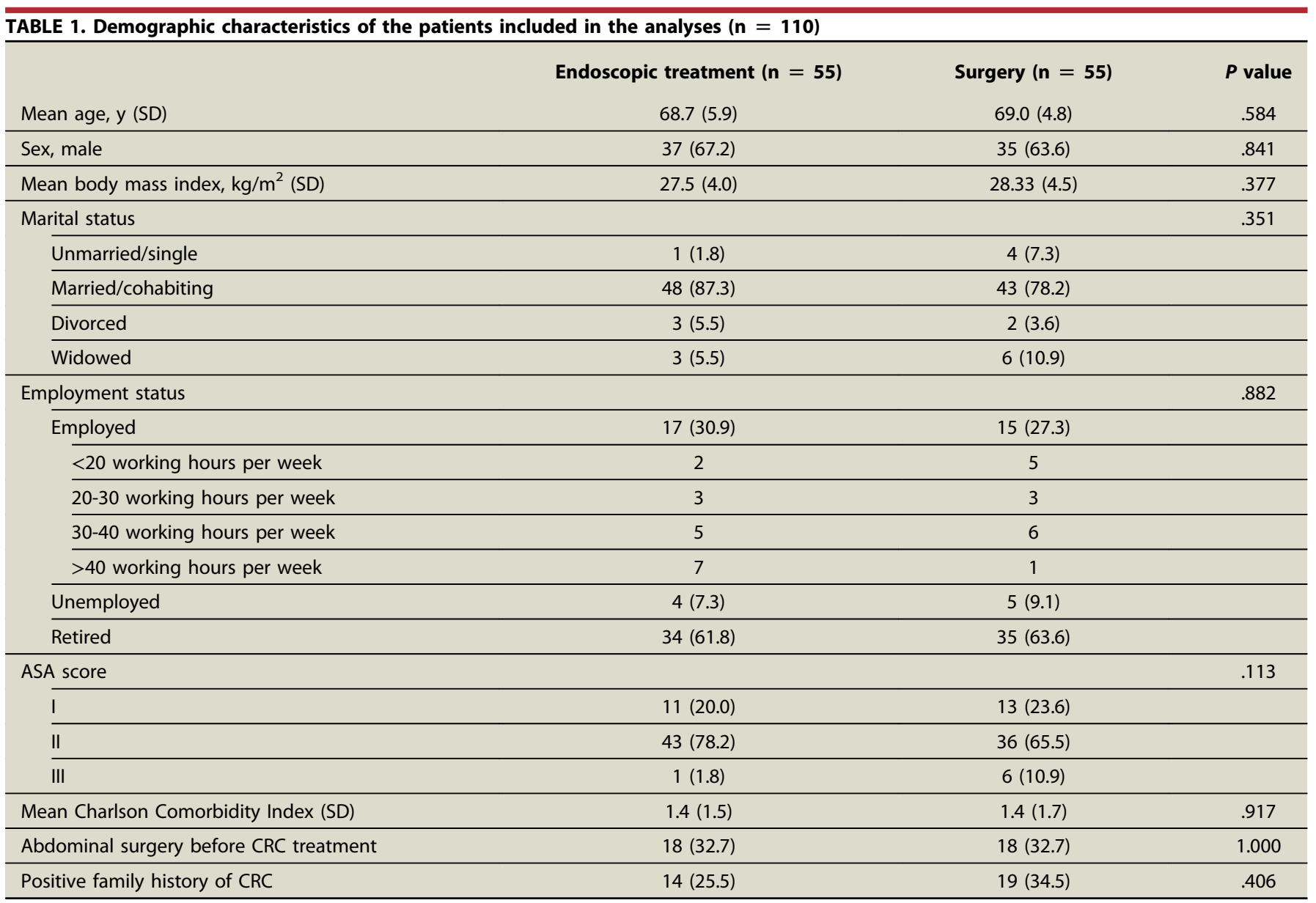

Values are $\mathrm{n}(\%)$ unless otherwise defined.

$A S A$, American Society of Anesthesiologists physical status classification system; $C R C$, colorectal cancer; $S D$, standard deviation.

influenced by the time between treatment and questionnaire completion because similar differences were observed in the short term (endoscopy, 14 days, vs surgery, 76 days; $P=$ .014 ) and long term (endoscopy, 26 days, vs surgery, 138 days; $P=.019)$ after treatment. The subgroup analysis with the separated surgery groups also yielded comparable results (endoscopy, 19.9 days, vs primary surgery, 142.9 days, $P<0.001$; and endoscopy, 19.9 days, vs secondary surgery, 78.6 days, $P=.011$ ).

\section{Sensitivity analysis}

The sensitivity analysis with all patients treated with TEM $(n=6)$ in the surgery group showed similar results on all study outcomes in all aforementioned analyses (data not shown; available on request).

\section{DISCUSSION}

Our study showed that the QoL (ie, global health status, functioning and severity of symptoms), as measured with the EORTC QLQ-C30 and EQ-5D-5L, was comparable in endoscopically and surgically treated patients. Yet, the perceived recovery time of endoscopically treated patients was 3 months shorter than that of the surgically treated patients. Furthermore, the endoscopy group did not report more fear of cancer recurrence than the surgery group. To our knowledge, this is the first study to provide empirical data on treatment impact from the T1 CRC patient's perspective.

The results of the CWS may have come as a surprise, because similarly designed studies of early-stage neoplasms in the upper GI tract (early gastric cancer ${ }^{43}$ and Barrett's esophagus ${ }^{36,44}$ ) reported that therapeutic endoscopy is associated with a significantly higher degree of fear of cancer recurrence than surgery. This difference was mainly attributed to the more intensive follow-up program, which was offered to endoscopically treated patients. In our study, however, the endoscopy group also received a strict follow-up consisting of multiple surveillance endoscopies, whereas the surgery group in general was followed up with occasional abdominal and chest imaging. We propose several other hypotheses that may have contributed to or explained this finding. First, 6 patients actively refused surgical treatment, despite it being recommended by their treating clinician. This could have 


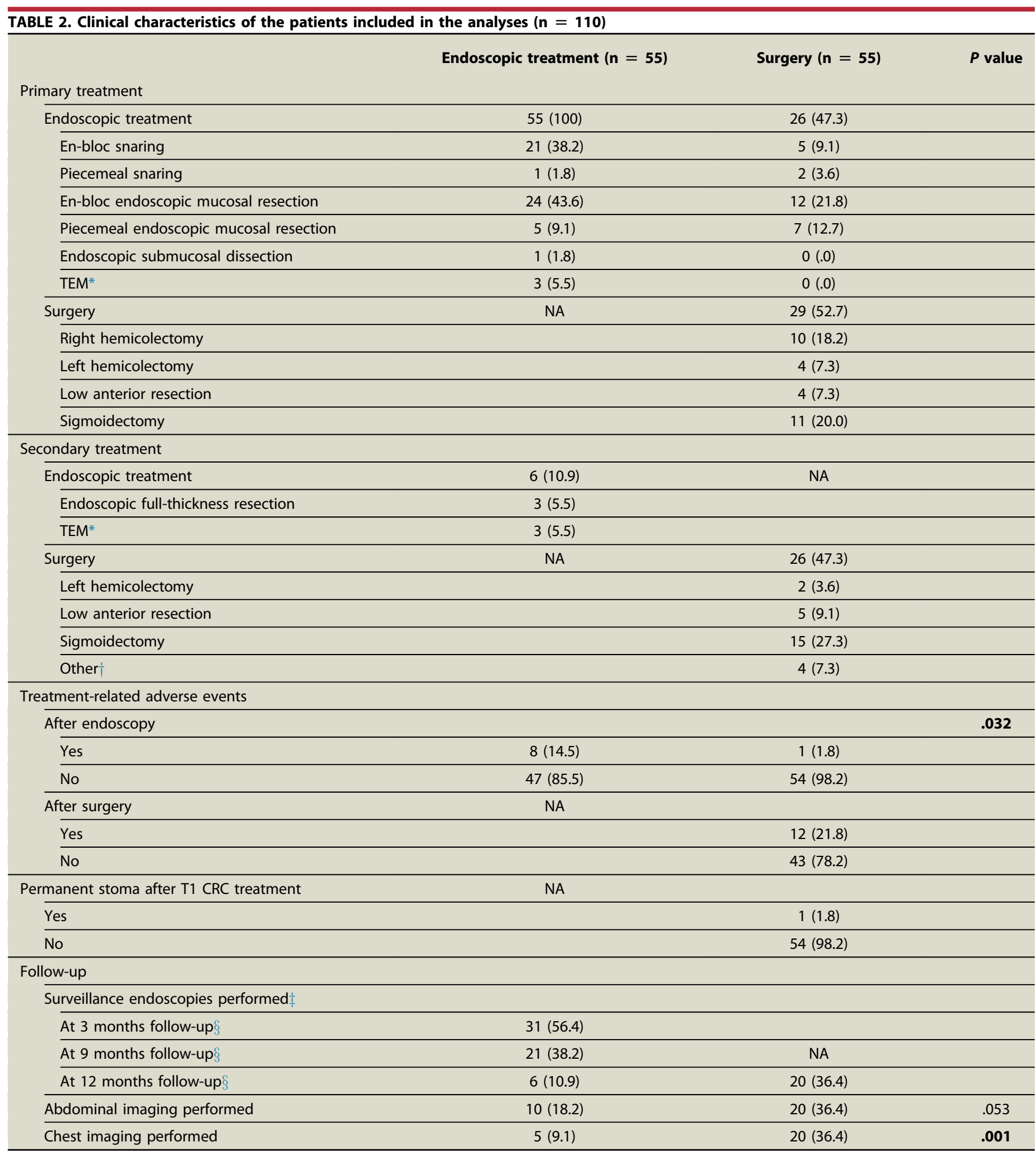

Values are $\mathrm{n}(\%)$. Significant values $(P<.05)$ are in bold.

$T E M$, Transanal endoscopic microsurgery; $N A$, not applicable; $T 1 C R C$, early invasive colorectal cancer.

*Patients treated with TEM underwent a local excision of the tumor without lymph node dissection and are therefore considered as endoscopically treated patients. $\dagger$ Tight-sided hemicolectomy $(n=1)$, rectosigmoid resection $(n=1)$, transverse resection $(n=1)$, and segmental resection of the colon descendens $(n=1)$.

$\ddagger$ After radical endoscopic resection of T1 CRC, the Dutch guidelines recommend to perform surveillance colonoscopies at 3 and 9 months of follow-up, followed by routine surveillance according to the guidelines after surgery for T1 CRC. After surgical resection of T1 CRC, the guidelines recommend to perform a surveillance colonoscopy 12 months of follow-up and then with an interval of 3-5 years. ${ }^{5}$

$\S$ With \pm 2 months margin. Only surveillance endoscopies scheduled according to the guidelines (ie, endoscopy group, first surveillance endoscopy at 3 months, second at 9 months, and third at 12 months; surgery group, first surveillance endoscopy at 12 months) are counted. 


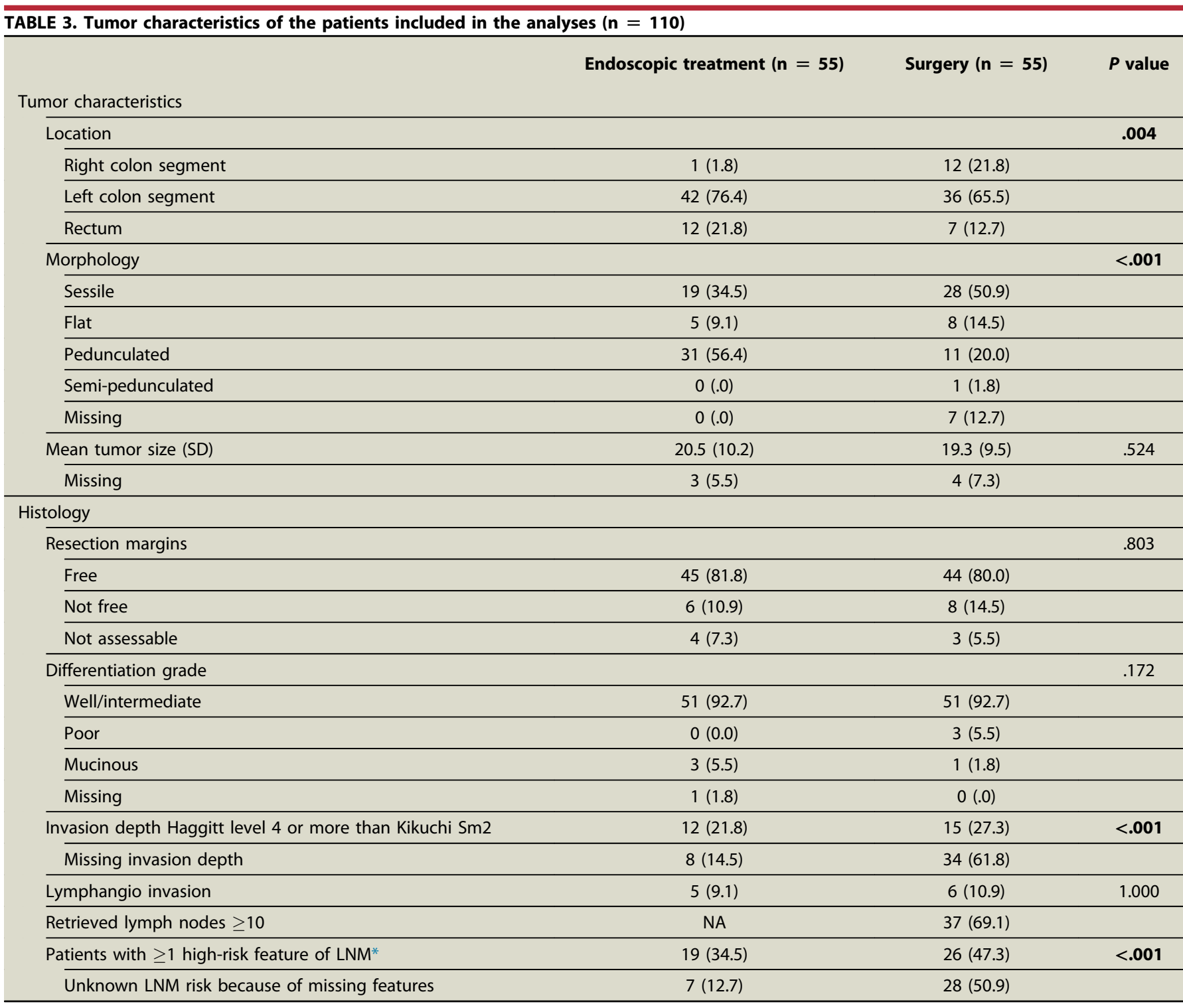

Values are $\mathrm{n}(\%)$ unless otherwise defined. Significant values $(P<.05)$ are in bold.

LNM, Lymph node metastasis; SD, standard deviation; NA, not applicable.

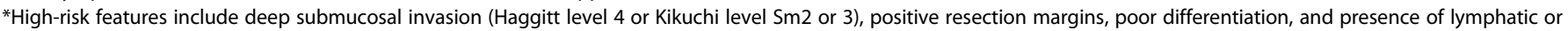
vascular invasion. Budding was not taken into account because the Dutch guideline does not recognize the presence of budding as a high-risk feature. ${ }^{5}$

led to less fear of cancer recurrence because these patients may have accepted the potential negative consequences of their decision. The relatively low CWS scores of these 6 patients also supported this hypothesis (median, 4.5; range, $0-9)$. Second, endoscopically treated T1 CRC patients may have a different view on the strict follow-up. Today, T1 CRC cases are often detected with population-based screening programs. ${ }^{1}$ Participants of such screening programs generally show more health-seeking behavior ${ }^{45}$ and may be comforted by frequent doctor visits and intensive surveillance. Third, surgery for T1 CRC may not lead to a greater sense of security than endoscopic treatment. For early gastric and esophageal cancer, it is believed that surgically treated patients had less fear of cancer recurrence because they were more likely to believe that all potential recurrence sites had been removed. ${ }^{36,43,44}$ However, this does not apply to T1 CRC patients, because surgical treatment for T1 CRC only involves a partial removal of the large bowel in general. ${ }^{2}$ In fact, depending on the timing of the resection, surgery may even cause more fear of cancer recurrence. Our subgroup analysis showed that primarily operated patients had significantly higher CWS scores than endoscopically treated patients, possibly because they had the feeling that their disease was so serious that it could not be treated endoscopically. However, when surgery is additionally performed after endoscopic tumor resection, patients do not report more fear of cancer recurrence. It seems that these patients are more likely to view their secondary surgery as an extra reassurance that their disease 


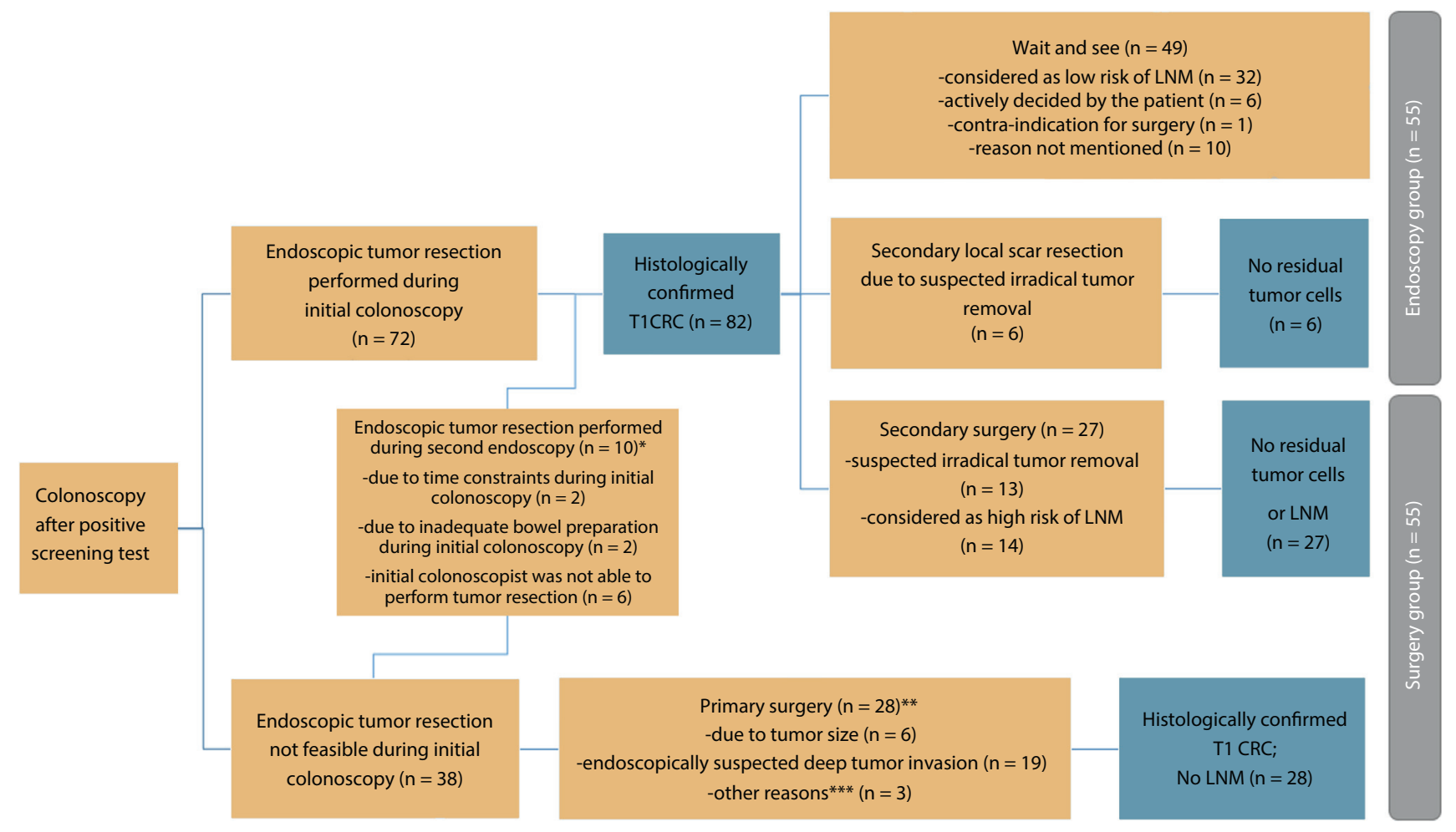

Figure 2. Overview of therapeutic decision-making of all T1 CRC patients who responded to the questionnaire $(\mathrm{n}=110)$.

*The median time interval between initial and second endoscopy was 43.5 days (interquartile range, 28-89). **The median time interval between initial colonoscopy and primary surgical resection was 34.5 days (interquartile range, 29-56).***Because of tumor location $(n=1)$, multiple polyps $(n=1)$, and high adverse event risk estimated if performing endoscopic resection $(\mathrm{n}=1)$.

$T 1 C R C$, Early invasive colorectal cancer; $L N M$, lymph node metastasis.

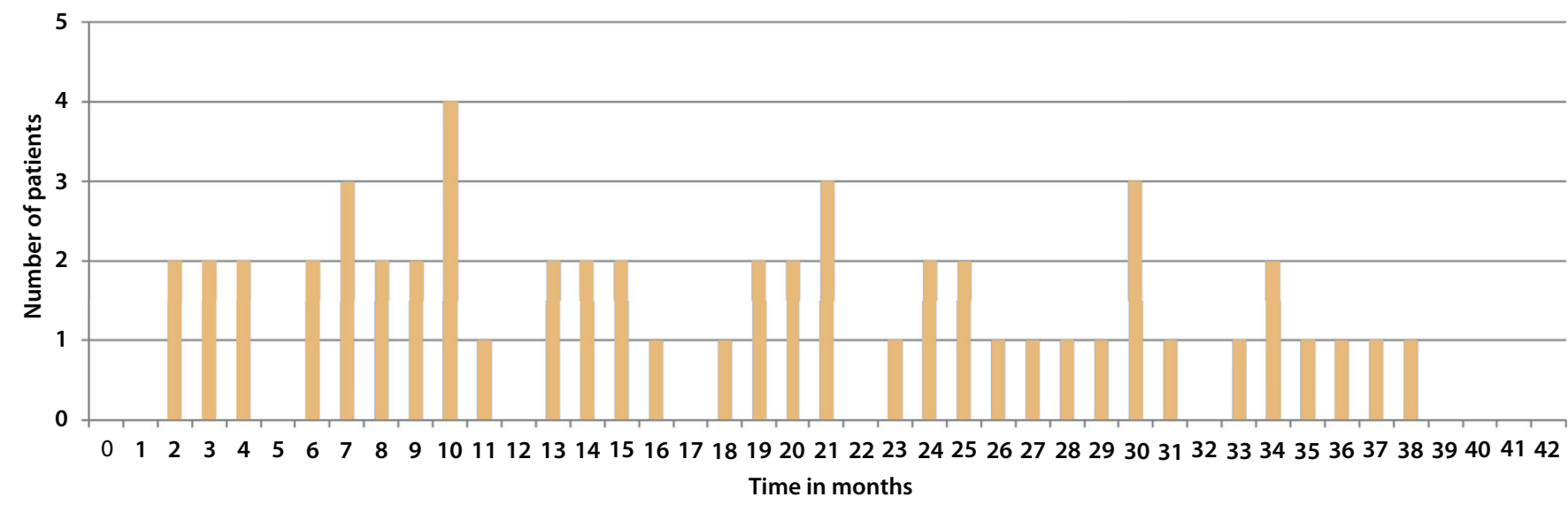

Figure 3. Time in months between treatment and moment of filling in the questionnaire, endoscopic treatment (median, 18; interquartile range, 9-26.5).

was adequately treated or cured. Last, an additional local scar resection was performed in 6 endoscopically treated patients: this additional therapy may have further reassured them about the curative potential of their endoscopic treatment. However, no support for this hypothesis can be found in their CWS scores (median, 9; range, 3-13).

In contrast to similar studies in early upper tract neoplasms, ${ }^{36,43,44}$ QoL did not favor endoscopic treatment compared with surgery in T1 CRC patients, despite that endoscopic resection is much less invasive. Instead, the faster recovery essentially favors endoscopic tumor resection from the patient's perspective.

Although self-perceived global QoL status (EuroQoL self-rated visual analogue scale and EORTC QLQ-C30 global health status scores) did not significantly differ, the shortterm societal value of patients' health state (EQ-5D score) of the endoscopy group was lower than that of the surgery group. Because this discrepancy between QoL from the 


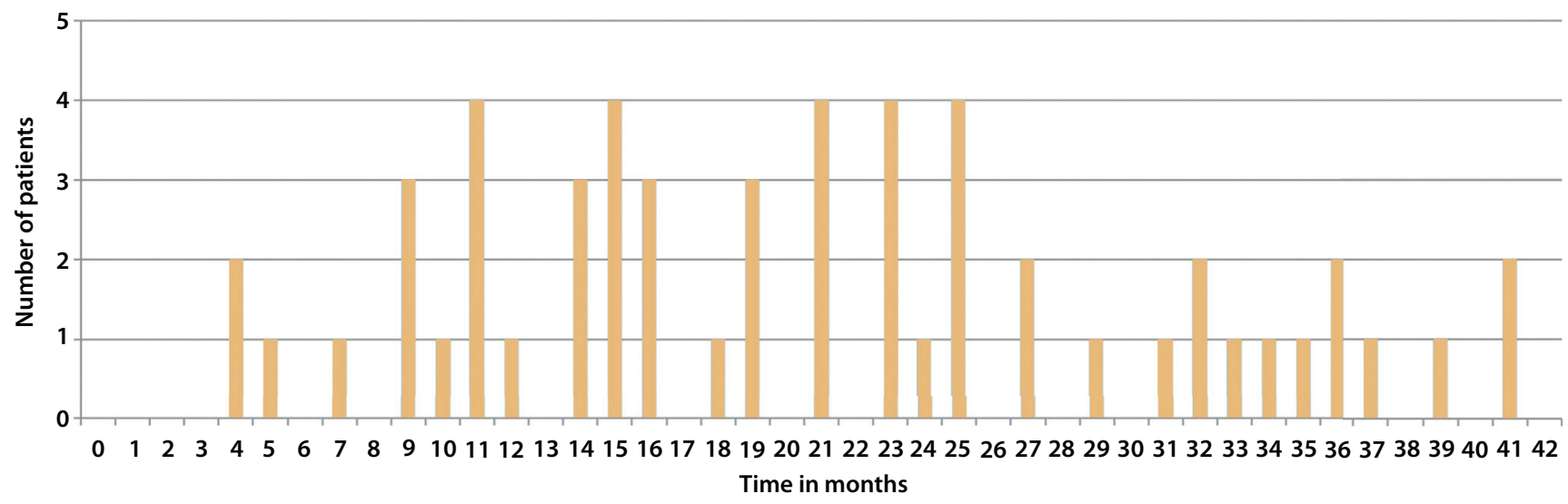

Figure 4. Time in months between treatment and moment of filling in the questionnaire, surgery (median, 21; interquartile range, 14-27).

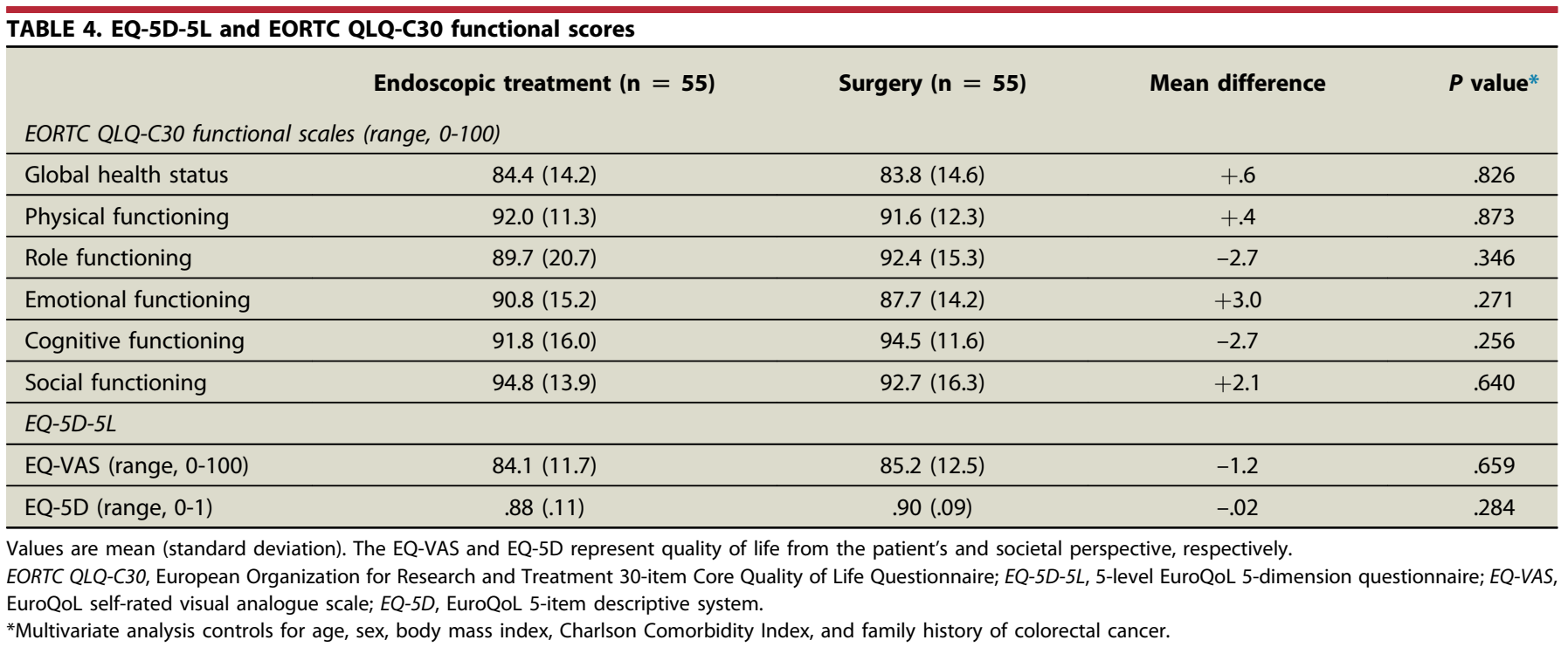

societal and the patient's perspective may indicate relatively lower health expectations in endoscopically treated patients, this may also explain the faster recovery perceived by them. However, despite statistical significance, the magnitude of the difference on the short-term EQ-5D was not considered to be clinically relevant (ie, $.07-.12$ points) ${ }^{46}$ suggesting that this explanation is unlikely. Alternatively, we believe that the faster recovery is more likely to be explained by the superior QoL directly after endoscopic treatment for T1 CRC. A prospective study comparing patient-reported QoL of endoscopic submucosal dissection with laparoscopic colectomy at 14 days after treatment concluded that endoscopic submucosal dissection provided a significantly better QoL than colectomy. ${ }^{47}$

However, such difference in QoL seems to disappear after a longer period of time. In our study, which had a median interval of 19 months between treatment and questionnaire completion, neither significant nor clinically relevant differences on the EORTC QLQ-C30 and EQ-5D-5L were found between the 2 treatment groups. These results corroborate 2 previous studies reporting that QoL of patients treated with total mesorectal excision or TEM was comparable from 1 year after treatment. ${ }^{48,49}$

Our hypothesis is that the impact of both surgical and endoscopic T1 CRC treatment does not exceed the range of patients' adaptability or resilience. In other words, a possible difference in treatment impact may only influence the QoL shortly after treatment. However, such influence may be masked after a longer period of time, when patients have adapted themselves to their new condition or have restored their QoL.

The clinical implications of our study results can mainly be sought in providing quantitative measures of relevant patient-reported outcomes. These can be integrated in the process of therapeutic decision-making, especially in the absence of a clear indication for surgical or endoscopic treatment. As we can deduce from our data, therapeutic decision-making in T1 CRC patients remains challenging in Dutch daily practice. For example, suspected 


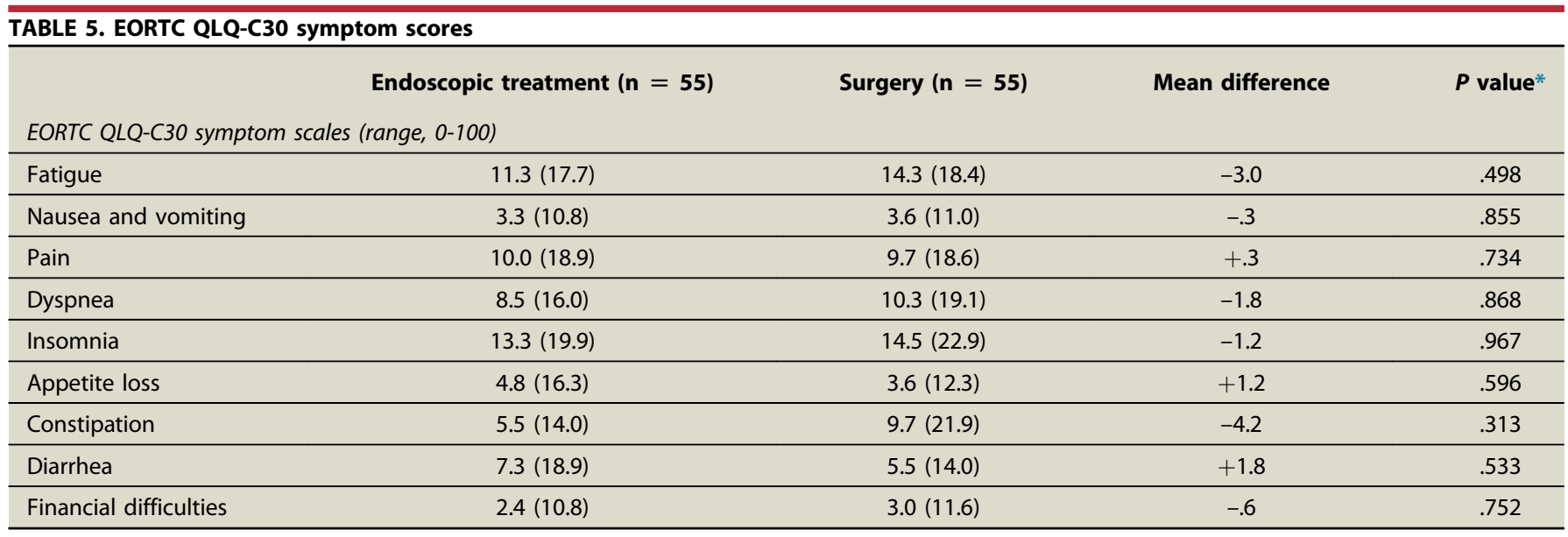

Values are mean (standard deviation).

EORTC QLQ-C30, European Organization for Research and Treatment 30-item Core Quality of Life Questionnaire.

*Multivariate analysis controls for age, sex, body mass index, Charlson Comorbidity Index, and family history of colorectal cancer.

TABLE 6. Fear of cancer recurrence and subjective time to recovery

\begin{tabular}{lccc}
\hline & Endoscopic treatment $(\mathbf{n}=\mathbf{5 5})$ & Surgery $(\mathbf{n}=\mathbf{5 5})$ & Mean difference \\
Cancer Worry Scale (range, 0-40) & $7.6(4.5)$ & $9.7(8.2)$ & -2.2 \\
\hline Patients' perceived time to recovery in days & $19.9(50.6)$ & $111.3(179.5)$ & -140 \\
\hline
\end{tabular}

Values are mean (standard deviation). Significant values $(P<.05)$ are in bold.

* Multivariate analysis controls for age, sex, body mass index, Charlson Comorbidity Index, and family history of colorectal cancer.

incomplete tumor removal led to an additional scar resection in 6 patients, whereas 13 other patients underwent surgery for the same reason. In most cases with a histologic high-risk feature in the endoscopy group, it was unclear why these patients did not receive an additional resection. Furthermore, all additional treatments actually performed after endoscopic resection did not provide additional oncologic benefit because no residual cancer cells or LNM were found. This illustrates the limitations of the current decision-making model in T1 CRC, which mainly focuses on preventing under-treatment but leads to a large amount of resections with suboptimal benefit. Given these limitations, we strongly stress the importance of eliciting the patient's perspective in therapeutic decision-making for T1 CRC.

Several strengths of our study merit attention. First, the overall response rate was relatively high $(92.4 \%)$, consequently minimizing the risk of selection bias. Second, all questionnaires included in this study have been validated and are widely used. Third, by including as many predefined confounders as possible, we reduced the possibility of confounding by indication and increased the likelihood that eventual outcome differences could be attributed to a specific treatment. Finally, this is the first study in T1 CRC patients that compares QoL, fear of cancer recurrence, and perceived time to recovery after endoscopic and surgical tumor resection.

Several limitations of this study should also be mentioned. First, the time between treatment and date of filling in the questionnaire varied considerably in this study population. Although the average interval did not significantly differ between the 2 treatment groups, we stratified our analyses by time between treatment and questionnaire completion to explore the influence of this important effect modifier on the study outcomes. Second, extrapolation of our results to other T1 CRC patients may be hampered because, for practical reasons, the study population exclusively consisted of participants of the Dutch Bowel Cancer Screening Programme. Because of the healthy user effect, ${ }^{50}$ screening-detected T1 CRC cases may not represent the average T1 CRC patient population. Third, for practical reasons, it was not possible to take into account the variety of treating physicians. This may have biased our results because personal characteristics and communication style of physicians, for example, can influence patients' health perceptions. Finally, and also most importantly, the cross-sectional study design limits the validity of our results. For example, it was not possible to measure QoL or fear of cancer recurrence of patients at baseline or prospectively at multiple time points in the follow-up. Given these limitations, we consider this study to be exploratory and as a starting point for prospective, longitudinal studies.

In summary, this study showed that endoscopically treated T1 CRC patients perceived 3 months faster recovery than surgically treated ones. Moreover, the endoscopy group did not report worse QoL or more fear of cancer recurrence than the surgery group. Because patient- 
reported outcomes are becoming increasingly important in cancer care, the results of this study contribute to the shared therapeutic decision-making process of clinicians and T1 CRC patients.

\section{ACKNOWLEDGMENTS}

We thank N. A. Osborne, J. M. Muller-van der Kolk (both from the department of Gastroenterology and Hepatology, Leiden University Medical Centre), A. H. Hekman, and H. J. Bertholee-Bosch (both from the department of Gastroenterology and Hepatology, Isala Hospital) for the logistical support and A. Farina Sarisqueta (Department of Pathology, Leiden University Medical Centre) for pathologic examination of the resected specimens.

\section{REFERENCES}

1. Arnold M, Sierra MS, Laversanne M, et al. Global patterns and trends in colorectal cancer incidence and mortality. Gut 2017;66:683-91.

2. Schmoll HJ, Van Cutsem E, Stein A, et al. ESMO Consensus Guidelines for management of patients with colon and rectal cancer. A personalized approach to clinical decision making. Ann Oncol 2012;23: 2479-516.

3. European Partnership Action Against Cancer consensus group; Borras JM, Albreht T, et al. Policy statement on multidisciplinary cancer care. Eur J Cancer 2014:50:475-80.

4. Yamamoto $\mathrm{S}$, Watanabe $M$, Hasegawa $H$, et al. The risk of lymph node metastasis in T1 colorectal carcinoma. Hepatogastroenterology 2004;51:998-1000.

5. Son HJ, Song SY, Lee WY, et al. Characteristics of early colorectal carcinoma with lymph node metastatic disease. Hepatogastroenterology 2008;55:1293-7.

6. Ha RK, Han KS, Sohn DK, et al. Histopathologic risk factors for lymph node metastasis in patients with T1 colorectal cancer. Ann Surg Treat Res 2017;93:266-71.

7. Miyachi H, Kudo SE, Ichimasa K, et al. Management of T1 colorectal cancers after endoscopic treatment based on the risk stratification of lymph node metastasis. J Gastroenterol Hepatol 2016;31: 1126-32.

8. Mou S, Soetikno R, Shimoda T, et al. Pathologic predictive factors for lymph node metastasis in submucosal invasive (T1) colorectal cancer: a systematic review and meta-analysis. Surg Endosc 2013;27:2692-703.

9. Watanabe T, Muro K, Ajioka Y, et al. Japanese Society for Cancer of the Colon and Rectum (JSCCR) guidelines 2016 for the treatment of colorectal cancer. Int J Clin Oncol 2018;23:1-34.

10. Bokey EL, Chapuis PH, Fung C, et al. Postoperative morbidity and mortality following resection of the colon and rectum for cancer. Dis Colon Rectum 1995;38:480-6; discussion 6-7.

11. Rickert A, Aliyev R, Belle $S$, et al. Oncologic colorectal resection after endoscopic treatment of malignant polyps: does endoscopy have an adverse effect on oncologic and surgical outcomes? Gastrointest Endosc 2014;79:951-60.

12. Paun BC, Cassie S, MacLean AR, et al. Postoperative complications following surgery for rectal cancer. Ann Surg 2010;251: 807-18.

13. Soung MC. Screening for cancer: when to stop? A practical guide and review of the evidence. Med Clin North Am 2015;99:249-62.

14. Singhal S, Changela K, Basi $P$, et al. Prescreening with FOBT improves yield and is cost-effective in colorectal screening in the elderly. ISRN Gastroenterol 2014;2014:179291.
15. Bronsgeest K, Huisman JF, Langers A, et al. Safety of endoscopic mucosal resection (EMR) of large non-pedunculated colorectal adenomas in the elderly. Int J Colorectal Dis 2017;32:1711-7.

16. Pontone S, Palma R, Panetta C, et al. Endoscopic mucosal resection in elderly patients. Aging Clin Exp Res 2017;29(Suppl 1):109-13.

17. Hashimoto R, Nakahori M, Matsuda T. The safety of diagnostic and therapeutic colonoscopies in the very elderly ( $>/=85$ years of age). Am J Gastroenterol 2016;111:1366-7.

18. King PM, Blazeby JM, Ewings $P$, et al. Randomized clinical trial comparing laparoscopic and open surgery for colorectal cancer within an enhanced recovery programme. Br J Surg 2006:93:300-8.

19. Guillou PJ, Quirke P, Thorpe H, et al. Short-term endpoints of conventional versus laparoscopic-assisted surgery in patients with colorectal cancer (MRC CLASICC trial): multicentre, randomised controlled trial. Lancet 2005;365:1718-26.

20. Weeks JC, Nelson H, Gelber S, et al. Short-term quality-of-life outcomes following laparoscopic-assisted colectomy vs open colectomy for coIon cancer: a randomized trial. JAMA 2002;287:321-8.

21. Braga M, Frasson M, Vignali A, et al. Laparoscopic vs. open colectomy in cancer patients: long-term complications, quality of life, and survival. Dis Colon Rectum 2005;48:2217-23.

22. Adachi Y, Sato K, Kakisako K, et al. Quality of life after laparoscopic or open colonic resection for cancer. Hepatogastroenterology 2003;50: 1348-51.

23. Janson M, Lindholm E, Anderberg B, et al. Randomized trial of healthrelated quality of life after open and laparoscopic surgery for colon cancer. Surg Endosc 2007;21:747-53.

24. Jayne DG, Guillou PJ, Thorpe H, et al. Randomized trial of laparoscopicassisted resection of colorectal carcinoma: 3-year results of the UK MRC CLASICC Trial Group. J Clin Oncol 2007;25:3061-8.

25. Lourenco T, Murray A, Grant A, et al. Laparoscopic surgery for colo rectal cancer: safe and effective? A systematic review. Surg Endosc 2008;22:1146-60

26. Niv Y, Bogolavski I, Ilani S, et al. Impact of colonoscopy on quality of life. Eur J Gastroenterol Hepatol 2012;24:781-6.

27. Kandel P, Wallace MB. Colorectal endoscopic mucosal resection (EMR) Best Pract Res Clin Gastroenterol 2017;31:455-71.

28. Bartel MJ, Brahmbhatt BS, Wallace MB. Management of colorectal T1 carcinoma treated by endoscopic resection from the Western perspective. Dig Endosc 2016;28:330-41.

29. Charlson ME, Pompei $P$, Ales $K L$, et al. A new method of classifying prognostic comorbidity in longitudinal studies: development and validation. J Chronic Dis 1987:40:373-83.

30. Herdman M, Gudex C, Lloyd A, et al. Development and preliminary testing of the new five-level version of EQ-5D (EQ-5D-5L). Qual Life Res 2011;20:1727-36.

31. Versteegh M, Vermeulen $K$, Evers $S$, et al. Dutch tariff for the five-level version of EQ-5D. Value Health 2016;19:343-52.

32. Aaronson NK, Ahmedzai S, Bergman B, et al. The European Organization for Research and Treatment of Cancer QLQ-C30: a quality-of-life instrument for use in international clinical trials in oncology. J Natl Cancer Inst 1993;85:365-76

33. Easterling DV, Leventhal $\mathrm{H}$. Contribution of concrete cognition to emotion: neutral symptoms as elicitors of worry about cancer. J Appl Psychol 1989;74:787-96.

34. Custers JA, van den Berg SW, van Laarhoven HW, et al. The Cancer Worry Scale: detecting fear of recurrence in breast cancer survivors. Cancer Nurs 2014;37:E44-50.

35. Custers JAE, Gielissen MFM, Janssen SHV, et al. Fear of cancer recurrence in colorectal cancer survivors. Support Care Cancer 2016;24 555-62.

36. Rosmolen WD, Boer KR, de Leeuw RJ, et al. Quality of life and fear of cancer recurrence after endoscopic and surgical treatment for early neoplasia in Barrett's esophagus. Endoscopy 2010;42:525-31

37. Rothman KJ. Epidemiology: an introduction. 2nd ed. New York, NY: Ox ford University Press; 2012. 
38. Wan GJ, Counte MA, Cella DF, et al. An analysis of the impact of demographic, clinical, and social factors on health-related quality of life. Value Health 1999;2:308-18.

39. Rodriguez JL, Hawkins NA, Berkowitz Z, et al. Factors associated with health-related quality of life among colorectal cancer survivors. Am J Prev Med 2015;49(6 Suppl 5):S518-27.

40. Bours MJ, van der Linden BW, Winkels RM, et al. Candidate predictors of health-related quality of life of colorectal cancer survivors: a systematic review. Oncologist 2016;21:433-52.

41. Sankar A, Johnson SR, Beattie WS, et al. Reliability of the American Society of Anesthesiologists physical status scale in clinical practice. Br J Anaesth 2014;113:424-32.

42. Barendse RM, van den Broek FJ, Dekker E, et al. Systematic review of endoscopic mucosal resection versus transanal endoscopic microsurgery for large rectal adenomas. Endoscopy 2011;43:941-9.

43. Choi JH, Kim ES, Lee YJ, et al. Comparison of quality of life and worry of cancer recurrence between endoscopic and surgical treatment for early gastric cancer. Gastrointest Endosc 2015;82:299-307.

44. Rosmolen WD, Nieuwkerk PT, Pouw RE, et al. Quality of life and fear of cancer recurrence after endoscopic treatment for early Barrett's neoplasia: a prospective study. Dis Esoph 2017;30:1-9.

45. Brookhart MA, Patrick AR, Dormuth C, et al. Adherence to lipidlowering therapy and the use of preventive health services: an investigation of the healthy user effect. Am J Epidemiol 2007;166:348-54.

46. Coretti S, Ruggeri M, McNamee P. The minimum clinically important difference for EQ-5D index: a critical review. Expert Rev Pharmacoecon Outcomes Res 2014;14:221-33.

47. Nakamura F, Saito Y, Haruyama S, et al. Short-term prospective questionnaire study of early postoperative quality of life after colorectal endoscopic submucosal dissection. Dig Dis Sci 2017;62:3325-35.

48. Doornebosch PG, Tollenaar RA, Gosselink MP, et al. Quality of life after transanal endoscopic microsurgery and total mesorectal excision in early rectal cancer. Colorectal Dis 2007;9:553-8.

49. Lezoche E, Paganini AM, Fabiani B, et al. Quality-of-life impairment after endoluminal locoregional resection and laparoscopic total mesorectal excision. Surg Endosc 2014;28:227-34.
50. Shrank WH, Patrick AR, Brookhart MA. Healthy user and related biases in observational studies of preventive interventions: a primer for physicians. J Gen Intern Med 2011;26:546-50.

51. Integraal Kankercentrum Nederland. Richtlijn Colorectaalcarcinoom (3.0), 2013 [updated April 16, 2014]. Available at: https://www. oncoline.nl/colorectaalcarcinoom. Accessed April 6, 2018.

Abbreviations: CWS, Cancer Worry Scale; EORTC QLQ-C30, European Organization for Research and Treatment 30-item Core Quality of Life Questionnaire; EQ-5D, EuroQoL 5-item descriptive system; EQ-5D-5L, 5 level EuroQoL 5-dimension questionnaire; EQ-5D, EuroQoL 5-item descriptive system; EQ-VAS, EuroQoL self-rated visual analogue scale; LNM, lymph node metastases; QoL, quality of life; T1 CRC, early invasive colorectal cancer; TEM, transanal endoscopic microsurgery.

DISCLOSURE: The following author disclosed financial relationships relevant to this publication: J. J. Boonstra: Consultant for Boston Scientific. All other authors disclosed no financial relationships relevant to this publication.

Copyright (C 2019 by the American Society for Gastrointestinal Endoscopy 0016-5107/\$36.00

https://doi.org/10.1016/j.gie.2018.09.026

Received June 14, 2018. Accepted September 13, 2018.

Current affiliations: Department of Gastroenterology and Hepatology (1), Department of Medical Decision Making (3), Department of Surgery (6), Department of Clinical Epidemiology (7), Leiden University Medical Centre, Leiden, The Netherlands; Department of Gastroenterology and Hepatology (2), Department of Surgery (4), Isala Hospital, Zwolle, The Netherlands; Department of Gastroenterology and Hepatology, University Medical Centre Utrecht, Utrecht, The Netherlands (5).

Reprint requests: Richard (Hao) Dang, BSc, Department of Gastroenterology and Hepatology, Leiden University Medical Centre, PO Box 9600, post zone C4-P, 2300 RC Leiden, The Netherlands.

\section{Registration of Clinical Trials}

Gastrointestinal Endoscopy follows the International Committee of Medical Journal Editors (ICMJE)'s Uniform Requirements for Manuscripts Submitted to Biomedical Journals. All clinical trials eventually submitted in GIE must have been registered through one of the registries approved by the ICMJE, and proof of that registration must be submitted to GIE along with the article. For further details and explanation of which trials need to be registered as well as a list of ICMJE-acceptable registries, please go to http://www.icmje.org. 